9th A. Friedmann International Seminar and

3rd Casimir Symposium 2015

International Journal of Modern Physics: Conference Series

Vol. 41 (2016) 1660131 (9 pages)

(C) The Author(s)

DOI: $10.1142 /$ S2010194516601319

\title{
Strong-field tests of $f(R)$-gravity in binary pulsars
}

Polina I. Dyadina

Sternberg Astronomical Institute, Lomonosov Moscow State University, Universitetsky Prospekt 13, Moscow 119991, Russia

Physics Department, Lomonosov Moscow State University, Moscow 119991, Russia guldur.anwo@gmail.com

Stanislav O. Alexeyev

Sternberg Astronomical Institute, Lomonosov Moscow State University, Universitetsky Prospekt 13, Moscow 119991, Russia alexeyev@sai.msu.ru

Salvatore Capozziello

Dipartimento di Scienze Fisiche, Universit di Napoli "Federico II", 80126, Napoli, Italy INFN Sezione di Napoli, 80126, Napoli, Italy

Gran Sasso Science Institute (INFN), Via F. Crispi 7, I-67100, L'Aquila, Italy capozzie@na.infn.it

Mariafelicia De Laurentis

Dipartimento di Scienze Fisiche, Universit di Napoli "Federico II", 80126, Napoli, Italy Tomsk State Pedagogical University, ul. Kievskaya 60, 634061 Tomsk, Russia mfdelaurentis@tspu.edu.ru

Kristina A. Rannu

Sternberg Astronomical Institute, Lomonosov Moscow State University, Universitetsky Prospekt 13, Moscow 119991, Russia melruin1986@gmail.com

Received 15 September 2015

Revised 4 October 2015

Published 18 March 2016

We develop the parameterized post-Keplerian approach for class of analytic $f(R)$-gravity models. Using the double binary pulsar system PSR J0737-3039 data we obtain restrictions on the parameters of this class of $f(R)$-models and show that $f(R)$-gravity is not

This is an Open Access article published by World Scientific Publishing Company. It is distributed under the terms of the Creative Commons Attribution 4.0 (CC-BY) License. Further distribution of this work is permitted, provided the original work is properly cited. 


\section{P. I. Dyadina et al.}

ruled out by the observations in strong field regime. The additional and more strong corresponding restriction is extracted from solar system data.

Keywords: Binary pulsars; $f(R)$-gravity; post-Keplerian formalism.

PACS numbers: 04.50.Kd, 04.70.-s, 04.80.Cc, 98.80.Es

\section{Introduction}

The theory of general relativity (GR) greatly improved the understanding of our Universe. It allows going beyond the Newtonian picture of the world. Anyway our present understanding has several shortcomings. A set of phenomena exists that we can not directly explain in the framework of GR. For explanation these phenomena many extended gravity models are created. One of the most perspective way to expand GR is to include additional corrections in terms of the Ricci scalar into the Lagrangian. This method underlies $f(R)$-gravity. ${ }^{1-3}$ This theory of gravity could allow to explain Dark Energy (DE) and Dark Matter (DM) by changing the geometry of a space-time and each scale demonstrates its specific geometry. This fact fully agrees with the early spirit of GR that could not act in the same way at all scales. ${ }^{4,5}$

However any theory of gravity should be verifiable. The discovery of binary pulsars PSR $1913+16$ by Hulse and Taylor in 1974 has opened a new testing ground for GR and its extensions. ${ }^{6}$ Before such discovery the only available testing ground for extended gravity was the Solar System where the gravitational field is relatively weak, slowly varying and representing a small perturbation of a flat space-time. It is important that in the case of binary pulsars one deals with a gravitational field which is stronger than in the case of the Solar System. Moreover, due to the high stability of the pulse arrival it is possible to observe the dynamics of the orbital motion with such an accuracy at which the effects of gravitational waves emission could appear. All this make the binary pulsars an indispensable laboratory for studying the behavior of different models of gravity. ${ }^{7,8}$

In this work we intend to use the data for the binary pulsar PSR J0737-3039. ${ }^{9,} 10$ PSR J0737-3039 is the only known double binary pulsar. The extraordinary closeness of the system components, small orbital period and the fact that we see almost edge-on system allow to investigate the manifestation of relativistic effects with the highest available precision.

The structure of the paper is the following. In Section 2 we develop the postKeplerian formalism for analytic $f(R)$-gravity models and obtain observational constraints arising from PSR J0737-3039 and Solar System data. The Section 3 contains our conclusions.

\section{2. $f(R)$-Gravity and Restriction Constraints}

\section{1. $f(R)$-gravity background}

The $f(R)$-gravity models are based on different expansions and enlargements of GR. Higher-order curvature invariants and minimally or non-minimally coupled 
scalar fields are added into the gravity action. In $f(R)$-gravity one considers a general function $f(R)$ instead of the Hilbert-Einstein Lagrangian $R$ that is linear with respect to the Ricci scalar $R$. The only assumption that we make is that $f(R)$ is an analytic function. The gravitational action now looks like: ${ }^{4,5,11,12}$

$$
S=\int d^{4} x \sqrt{-g}\left[f(R)+\kappa L_{m}\right]
$$

where $\kappa=16 \pi G / c^{4}$ is the coupling, $g$ is the determinant of the metric and $L_{m}$ is the standard matter contribution.

We assume that the $f(R)$ Lagrangian is analytic (i.e. Taylor expandable) in terms of the Ricci scalar, therefore: ${ }^{11,12}$

$$
f(R)=\sum_{n} \frac{f^{n}\left(R_{0}\right)}{n !}\left(R-R_{0}\right)^{n} \simeq f_{0}+f_{0}^{\prime} R+\frac{1}{2} f_{0}^{\prime \prime} R^{2}+\ldots,
$$

where

$$
f_{0}=\text { const }, \quad f_{0}^{\prime}=\left.\frac{d f(R)}{d R}\right|_{R=0}, \quad f_{0}^{\prime \prime}=\left.\frac{d^{2} f(R)}{d R^{2}}\right|_{R=0} .
$$

The flat background is recovered for $R=R_{0} \simeq 0$. GR is recovered in the limit $f_{0}=0, f_{0}^{\prime}=4 / 3, f_{0}^{\prime \prime}=0 .{ }^{4,5}$ Hereafter we assume $f_{0}=0 .{ }^{4,5}$

\subsection{Restrictions from Solar system}

Now we can start from considering $f(R)$-gravity in the weak field limit, namely in the framework of the PPN formalism. ${ }^{13-16}$

Drawing an analogy between the scalar-tensor gravity and the higher order theories of gravity, Capozziello and Troisi ${ }^{11,12}$ developed the PPN formalism application to $f(R)$-gravity. The similarity between the non-minimally coupled scalar models (of Brans-Dicke type ${ }^{17,18}$ ) and with higher order curvature corrections have been discussed since 1983. ${ }^{19}$ Using the results of Capozziello and Troisi, we carried out the Eddington's parameters for the considered model of $f(R)$-gravity in terms of the parameters $f_{0}^{\prime}$ and $f_{0}^{\prime \prime}$ :

$$
\begin{aligned}
& \gamma_{R}^{P P N}-1=-\frac{\left(f_{0}^{\prime \prime}\right)^{2}}{f_{0}^{\prime}+2\left(f_{0}^{\prime \prime}\right)^{2}}, \\
& \beta_{R}^{P P N}-1=\frac{1}{4} \frac{f_{0}^{\prime}\left(f_{0}^{\prime \prime}\right)^{4}}{2\left(f_{0}^{\prime}\right)^{3}+20 f_{0}^{\prime}\left(f_{0}^{\prime \prime}\right)^{4}+11\left(f_{0}^{\prime}\right)^{2}\left(f_{0}^{\prime \prime}\right)^{2}+12\left(f_{0}^{\prime \prime}\right)^{6}} .
\end{aligned}
$$

We know that GR works in the Solar System with the highest precision so we can fix $f_{0}^{\prime}=4 / 3$ and using the observational values of parameters $\gamma^{P P N}, \beta^{P P N}$ (see. Table 1$),{ }^{20}$ we obtain the limitation on the value of $f_{0}^{\prime \prime}$ by solving the system (4):

$$
\begin{gathered}
\gamma^{P P N}: \quad-0.0055 \leq f_{0}^{\prime \prime} \leq 0, \\
\beta^{P P N}: \quad-0.19 \leq f_{0}^{\prime \prime} \leq 0 .
\end{gathered}
$$


Table 1. Physical meanings and experimental values of PPN parameters

\begin{tabular}{ccc}
\hline $\begin{array}{c}\text { PPN } \\
\text { parameter }\end{array}$ & Physical meaning & $\begin{array}{c}\text { Experimental } \\
\text { value }\end{array}$ \\
\hline$\gamma$ & $\begin{array}{c}\text { space-curvature } \\
\text { produced by unit restmass } \\
\text { nonlinearity in } \\
\beta\end{array}$ & $1 \pm 2.3 \times 10^{-5}$ \\
& $\begin{array}{c}\text { the superposition law } \\
\text { for gravity }\end{array}$ & \\
\hline
\end{tabular}

It is important to note that we use a down limit for $\gamma^{P P N}$ and top for $\beta^{P P N}$ values because $f(R)$ gravity predicts that $\gamma^{P P N}<1$ and $\beta^{P P N}>1$. In the case $\gamma^{P P N}>1$ and $\beta^{P P N}<1$ value of $f_{0}^{\prime \prime}$ becomes an imaginary quantity, becoming completely unphysical.

\subsection{The parameterized post-Keplerian formalism}

The parameterized post-Keplerian formalism (PPK) was developed by Damour and Deruelle $^{21}$ and further improved by Damour and Taylor. ${ }^{22}$ It was created to link the arrival time of pulses and their radiation time in the frame of the pulsar. Damour et al. have showed that all the independent $O\left(v^{2} / c^{2}\right)$ timing effects can be represented in a simple mathematical way that is generic for a wide class of extended gravities. PPK is a strong-field analogue of the PPN formalism. It includes such effects as the Einstein time delay, Römer time delay, Shapiro time delay and the effects of aberration. The general form of these corrections is model-independent, therefore all possible manifestations of the extended gravity model can be expressed through the 8 PPK parameters $\dot{\omega}, \gamma, \dot{P}_{b}, r, s, \delta_{\theta}, \dot{e}, \dot{x}$. However, we consider only the most accurately measured parameters, so we do not take into account the last three of them. In this work we obtain the analytical form of four PPK parameters:

$$
\begin{aligned}
\dot{\omega}= & \left(\frac{2 \pi}{P_{b}}\right)^{5 / 3} \frac{G^{2 / 3} M_{\odot}^{2 / 3}\left(m_{1}+m_{2}\right)^{2 / 3}}{c^{2}\left(1-e^{2}\right)}\left(\frac{2\left(f_{0}^{\prime}\right)+4\left(f_{0}^{\prime \prime}\right)^{2}}{2\left(f_{0}^{\prime}\right)+3\left(f_{0}^{\prime \prime}\right)^{2}}\right)^{2 / 3} \\
& \times\left(\frac{7}{2}-\frac{2\left(f_{0}^{\prime \prime}\right)^{2}}{f_{0}^{\prime}+2\left(f_{0}^{\prime \prime}\right)^{2}}-\frac{1}{2}\left(\frac{4\left(f_{0}^{\prime}\right)^{3}+24\left(f_{0}^{\prime \prime}\right)^{6}+22\left(f_{0}^{\prime}\right)^{2}\left(f_{0}^{\prime \prime}\right)^{2}+41 f_{0}^{\prime}\left(f_{0}^{\prime \prime}\right)^{4}}{4\left(f_{0}^{\prime}\right)^{3}+24\left(f_{0}^{\prime \prime}\right)^{6}+22\left(f_{0}^{\prime}\right)^{2}\left(f_{0}^{\prime \prime}\right)^{2}+40 f_{0}^{\prime}\left(f_{0}^{\prime \prime}\right)^{4}}\right)\right. \\
& \left.\times\left[\frac{2 f_{0}^{\prime}+3\left(f_{0}^{\prime \prime}\right)^{2}}{2 f_{0}^{\prime}+4\left(f_{0}^{\prime \prime}\right)^{2}}\right]^{2}\right), \\
\gamma= & \left(\frac{2 \pi}{P_{b}}\right)^{-1 / 3} \frac{G^{2 / 3} M_{\odot}^{2 / 3} m_{2}}{c^{2}\left(m_{1}+m_{2}\right)^{4 / 3}}\left(m_{1}+m_{2}\left[2+\frac{\left(f_{0}^{\prime \prime}\right)^{2}}{2 f_{0}^{\prime}+3\left(f_{0}^{\prime \prime}\right)^{2}}\right]\right) \\
& \times\left(1+\frac{\left(f_{0}^{\prime \prime}\right)^{2}}{2 f_{0}^{\prime}+3\left(f_{0}^{\prime \prime}\right)^{2}}\right)^{-1 / 3},
\end{aligned}
$$




$$
\begin{aligned}
& r=\frac{1}{4 c^{3}} G M_{\odot} m_{2}, \\
& s=\left(\frac{2 \pi}{P_{b}}\right)^{2 / 3} \frac{c x\left(m_{1}+m_{2}\right)^{2 / 3}}{\left(G M_{\odot}\right)^{1 / 3} m_{2}}\left(1+\frac{\left(f_{0}^{\prime \prime}\right)^{2}}{2 f_{0}^{\prime}+3\left(f_{0}^{\prime \prime}\right)^{2}}\right)^{-1 / 3} .
\end{aligned}
$$

The expression for the last parameter $\dot{P}_{b}$ was obtained by Capozziello and De Laurentis $^{4,5}$ in the form:

$$
\begin{aligned}
\dot{P}_{b}= & -\frac{6 \pi}{20}\left(\frac{2 \pi}{P_{b}}\right)^{5 / 3} \frac{\left(G M_{\odot}\right)^{5 / 3}}{c^{5}\left(1-e^{2}\right)^{7 / 2}} \frac{m_{1} m_{2}}{\left(m_{1}+m_{2}\right)^{1 / 3}} \\
& \times\left(\left(f_{0}^{\prime}\left(37 e^{4}+292 e^{2}+96\right)-\frac{f_{0}^{\prime \prime} \pi^{2}}{2 P_{b}\left(1+e^{2}\right)^{3}}\right.\right. \\
& \left.\times\left(891 e^{8}+28016 e^{6}+82736 e^{4}+43520 e^{2}+3072\right)\right) .
\end{aligned}
$$

\subsection{Restrictions from PSR J0737-3039}

Now we can proceed directly to the method of testing models of gravity ${ }^{22}$ and our additions. We plot the curves representing post-Keplerian parameters on the plane, in the $Y$-axis we put the allowed values of the companion masses $m_{2}$ and in the $X$-axis we put possible values of the pulsar $m_{1}$ masses. The region of all curves intersection within the measured accuracy displays the possible range of the pulsar's mass and its companion. For the appropriate gravity model all the curves of post-Keplerian parameters should "meet" at one point (taking into account the accuracy). The measurements of $n$ post-Keplerian parameters give $n-2$ tests. In our case there are $6-2=4$ ones.

Here it is importantly to point out that all the curves for PSR J0737-3039 intersect within the measurement accuracy in the GR case (see Fig. 1).
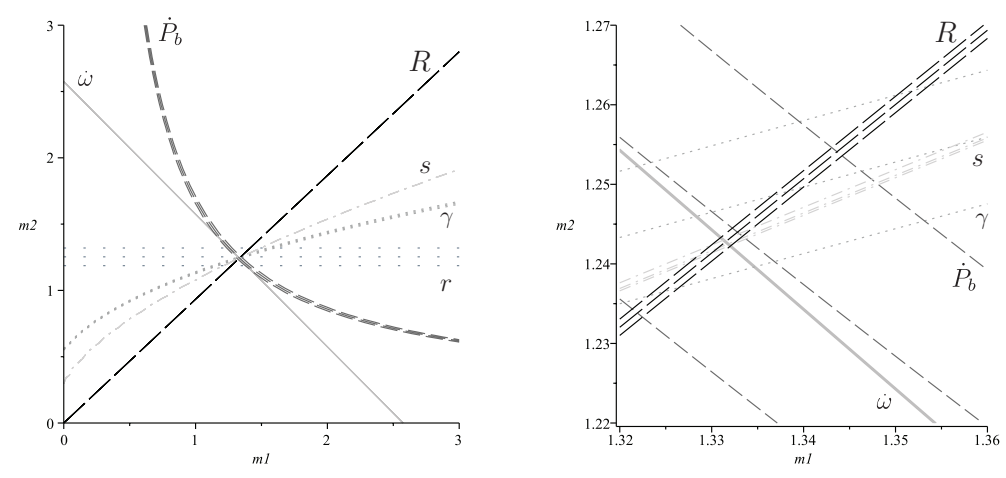

Fig. 1. Dependence of the companion mass upon the pulsar one at different scales for GR, $f_{0}^{\prime}=$ $4 / 3$ and $f_{0}^{\prime \prime}=0$. 
Table 2. Parameters PSR J0737-3039.

\begin{tabular}{ccc}
\hline Parameter & Physical meaning & Experimental value \\
\hline$P_{b}($ day $)$ & orbital period & $0.10225156248(5)$ \\
$e$ & eccentricity & $0.0877775(9)$ \\
$x(s)$ & projected semimajor axis & $1.415032(1)$ \\
$\dot{\omega}($ deg/yr $)$ & of the pulsar orbit & $16.89947(68)$ \\
$\tilde{\gamma}(m s)$ & secular advance & $0.3856(26)$ \\
$\dot{P}_{b}$ & of the periastron & time dilation parameter \\
$s$ & secular change of & $-1.252(17) \times 10^{-12}$ \\
$r(\mu s)$ & the orbital period & $6.21(33)$ \\
$R=\frac{m_{1}}{m_{2}}=\frac{x_{2}}{x_{1}}$ & Shapiro delay parameter & $0.99974(-39,+16)$ \\
\hline
\end{tabular}

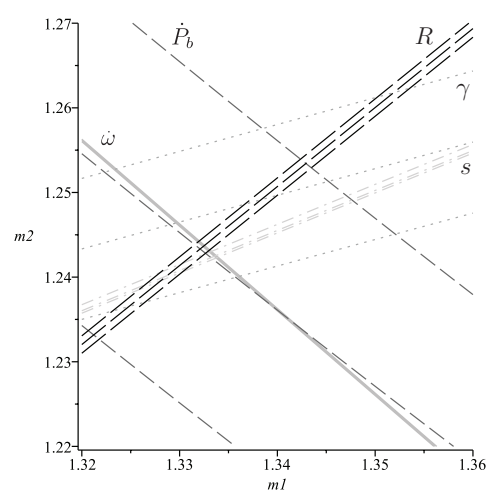

Fig. 2. Dependence of the companion mass upon the pulsar one, $f_{0}^{\prime}=4 / 3$ and $f_{0}^{\prime \prime}=-0.0623$. The last point of intersection $s\left(m_{1}, m_{2}\right), R\left(m_{1}, m_{2}\right)$ and $\dot{\omega}\left(m_{1}, m_{2}\right)$ within the measurement accuracy, when $f_{0}^{\prime}$ is fixed.

To obtain restrictions for $f(R)$-gravity we solve the system (6) and (7) considering observational data within the measurement accuracy for PSR J0737-3039 (see Table 2). In the first step we fix $f_{0}^{\prime}=4 / 3$ and we obtain that the curves intersect only when $f_{0}^{\prime \prime}$ takes the following values (see Fig. 2):

$$
-0.0623 \leq f_{0}^{\prime \prime} \leq 0
$$

In the second step we don't make any assumptions about values $f_{0}^{\prime}$ and $f_{0}^{\prime \prime}$. We solve the system (6) and (7) and the fact that we take into account parameter $\dot{P}_{b}$ allows to obtain the admitted region for $f_{0}^{\prime}$ besides restrictions for $f_{0}^{\prime \prime}$ (see Fig. 3 ):

$$
\begin{aligned}
1.317 & \leq f_{0}^{\prime} \leq 1.353, \\
-0.0627 & \leq f_{0}^{\prime \prime} \leq 0 .
\end{aligned}
$$




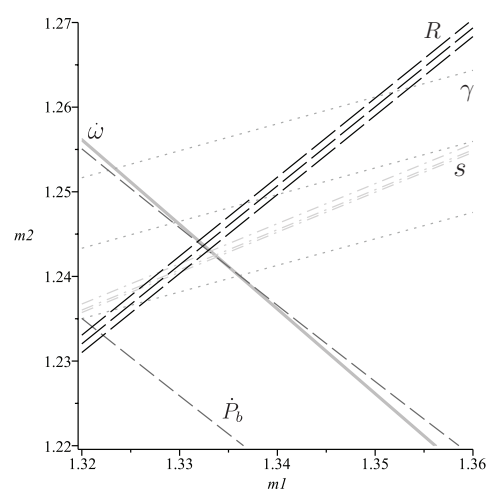

Fig. 3. Dependence of the companion mass upon the pulsar one, $f_{0}^{\prime}=1.353$ and $f_{0}^{\prime \prime}=-0.0627$. The last point of intersection $s\left(m_{1}, m_{2}\right), R\left(m_{1}, m_{2}\right)$ and $\dot{\omega}\left(m_{1}, m_{2}\right)$ within the measurement accuracy, when $f_{0}^{\prime}$ is not fixed.

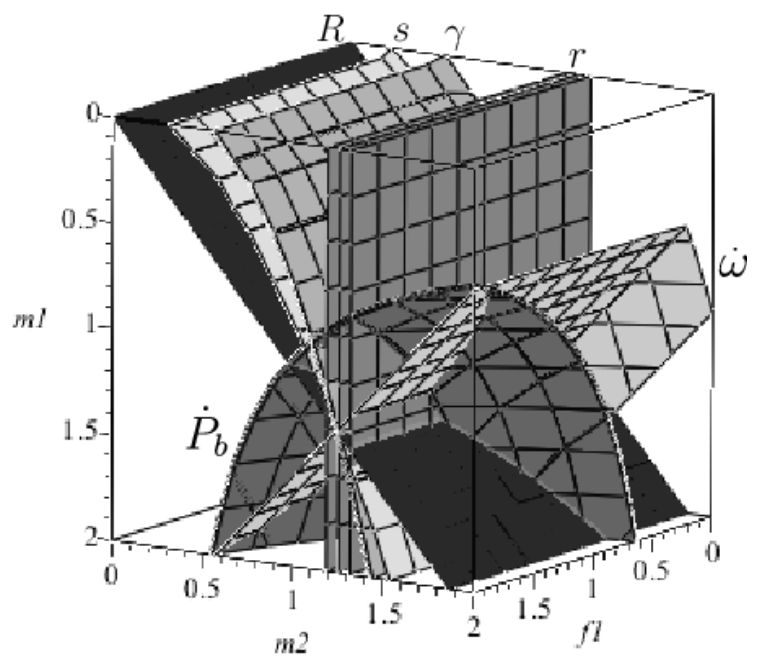

Fig. 4. Dependence of the parameter $f_{0}^{\prime}$ upon the pulsar and its companion masses.

In this case we construct the surfaces representing post-Keplerian parameters in the three-dimensional space, in the $Y$-axis we put the allowed values of the companion masses $m_{2}$, in the $X$-axis we put possible values of the pulsar $m_{1}$ masses and in the $Z$-axis we put the allowed values of the parameter $f_{0}^{\prime}$, which can be restrict by $\dot{P}_{b}$ surface (see Fig. 4 ).

In the case of GR when $f_{0}^{\prime}=4 / 3, f_{0}^{\prime \prime}=0$ we have the following masses of the pulsar system components:

$$
\begin{aligned}
& m_{p 1}=1.3381, \\
& m_{p 2}=1.2489 .
\end{aligned}
$$




\section{P. I. Dyadina et al.}

In the case of $f(R)$-gravity considering in the last point of intersection $f_{0}^{\prime}=$ $1.353, f_{0}^{\prime \prime}=-0.0627$ these quantities take the following values:

$$
\begin{aligned}
& m_{p 1}=1.3331 \\
& m_{p 2}=1.2429 .
\end{aligned}
$$

Hence the difference between GR and $f(R)$-gravity is $0.5 \%$.

\section{Conclusions}

We consider the analytical $f(R)$-gravity in the system of binary pulsars. To find the observational limitations on these models the PPK parameters and other orbital data of the double binary pulsar PSR J0737-3039 ${ }^{9,10}$ are applied. We find the analytical form of the four PPK parameters for the analytic $f(R)$-gravity. It is important that we use the general form of $f(R)$-gravity. The only assumption is that $f(R)$ is supposed to be an analytical function.

If one knows exactly the type of Lagrangian of considered system, the values of PPK parameters can be easily predicted and compared with observational constraints. In this work to get estimations for the $f_{0}^{\prime}$ and $f_{0}^{\prime \prime}$ we proceed the inverse process. So, the parameters $\dot{\omega}, \tilde{\gamma}, r, s$ are obtained as functions of $f_{0}^{\prime}$ and $f_{0}^{\prime \prime}$. We consider two cases. In the first case the value of $f_{0}^{\prime}$ is fixed and we obtain the bounds only for $f_{0}^{\prime \prime}$ :

$$
-0.0623 \leq f_{0}^{\prime \prime} \leq 0
$$

Then we go further and we find the admitted region of values for $f_{0}^{\prime}$ and $f_{0}^{\prime \prime}$ :

$$
\begin{aligned}
1.317 & \leq f_{0}^{\prime} \leq 1.353 \\
-0.0627 & \leq f_{0}^{\prime \prime} \leq 0 .
\end{aligned}
$$

The limits on $f_{0}^{\prime \prime}$ from the Solar System data are also established in our research. We apply the developments of Capozziello and Troisi ${ }^{11,12}$ for the analytic $f(R)$ gravity models. Our analysis shows that the observations in the Solar System (PPN) give tighter restrictions on $f_{0}^{\prime \prime}$ from parameter $\gamma^{P P N}$ :

$$
\gamma^{P P N}: \quad-0.0055 \leq f_{0}^{\prime \prime} \leq 0
$$

This apparent huge difference in the bounds may be explained invoking an underlying "chameleon" effect. ${ }^{23}$ This in turn would cause $f_{0}^{\prime \prime}$ to vary depending on the characteristic length scale or density of the system under consideration.

Thus Extended Theories of Gravity namely the analytic $f(R)$-gravity models are not excluded in strong and weak field regimes. This result gives rise for further studies of $f(R)$-gravity and other models based of such type of GR extension: Palatini $f(R)$-gravity, ${ }^{24}$ hybrid metric-Palatini $f(R)$-gravity ${ }^{25}$ and so on. 


\section{References}

1. S. Capozziello and R. de Ritis, Class. Quantum Grav. 11, 107 (1994); S. Capozziello and R. de Ritis, Phys. Lett. A 177, 1 (1993).

2. S. Capozziello, R. de Ritis and C. Rubano, Phys. Lett. A 177, 8 (1993).

3. S. Capozziello, M. Demianski, R. de Ritis and C. Rubano, Phys. Rev. D 52 (1995).

4. M. De Laurentis and S. Capozziello, Astrop. Phys. 35, 257 (2011).

5. M. De Laurentis and I. de Martino MNRAS D 741, 431 (2013).

6. R. A. Hulse and J. H. Taylor, Astrophys. J. Lett. 195, L51 (1975).

7. C. M. Will, Theory and Experiment in Gravitational Physics (Cambridge University Press, New York, 1981); Living Rev. Relativity 17, 4 (2014).

8. D. M. Eardley, Astrophys. J. Lett. 196, L59 (1975).

9. M. Burgay et al., Nature 426, 531 (2003).

10. M. Kramer et al., Science 341, 97 (2006).

11. S. Capozziello and A. Troisi, Phys. Rev. D 72, 044022 (2005).

12. S. Capozziello, A. Stabile and A. Troisi, Mod. Phys. Lett. A 21, 2291 (2006); S. Capozziello, A. Stabile and A. Troisi, Phys. Rev. D 76, 104019 (2007); S. Capozziello, A. Stabile and A. Troisi, Class. Quant. Grav. 25, 085004 (2008); S. Capozziello, A. Stabile and A. Troisi, Mod. Phys. Lett. A 24, 659 (2009); S. Capozziello, A. Stabile and A. Troisi, Int. Jour. of Theor. Phys. 49, 1251 (2010).

13. A. S. Eddington, The Mathematical Theory of Relativity (Cambridge University Press, London, 1922).

14. K. Nordtvedt, Phys. Rev. 169, 1017 (1968).

15. C. M. Will, Astrophys. J. 163, 611 (1971).

16. C. M. Will and K. Nordtvedt, Astrophys. J. 177, 757 (1972).

17. C. Brans and H. Dicke, Phys. Rev. 124, 925 (1961).

18. D. A. Tretyakova, A. A. Shatskiy, I. D. Novikov and S. O. Alexeyev, Phys. Rev. D 85, 124059 (2012).

19. P. Teyssandier and P. Tourranc, J. Math. Phys. 24, 2793 (1983).

20. S. G. Turyshev, Ann. Rev. Nucl. Part. Sci 58, 207 (2008).

21. T. Damour and N. Deruelle, Ann. Inst. Henri Poincare A 43, 107 (1985); Ann. Inst. Henri Poincare A 44, 263 (1986).

22. T. Damour and J. H. Taylor, Phys. Rev. D 45, 1840 (1992).

23. T. Faulkner, M. Tegmark, E. F. Bunn and Y. Mao, Phys. Rev. D 76, 063505 (2007).

24. G. J. Olmo, Int. J. Mod. Phys. D 20, 413 (2011).

25. S. Capozziello, T. Harko, T. S. Koivisto, F. S. N. Lobo and G. J. Olmo, JCAP 1304, 011 (2013). 